Supporting Information for

\title{
Structure, function, and thermodynamics of lactate dehydrogenases from humans and the malaria parasite $P$. falciparum
}

\author{
Sergei Khrapunov*1, Akiba Waterman ${ }^{2}$, Rudra Persaud ${ }^{2}$ and \\ Eric P. Chang ${ }^{2}$ \\ 1 - Department of Biochemistry, Albert Einstein College of Medicine, 1300 Morris Park \\ Avenue, Bronx, NY 10461 \\ 2 - Department of Chemistry and Physical Sciences, Pace University, 1 Pace Plaza, \\ New York, NY 10038 \\ *Correspondence to Sergei Khrapunov: sergei.khrapunov@einsteinmed.org
}

Supporting information includes 3 figures. 


\section{Supplementary Figures}

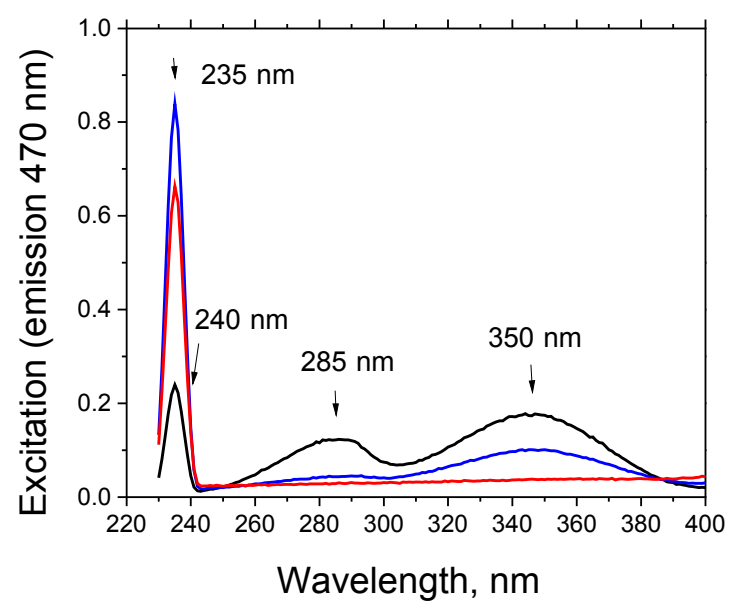

Figure S1. Excitation spectrum (Emission $470 \mathrm{~nm}$ ) of the binary complex of LDH from pig heart (phLDH) and NADH at $20^{\circ} \mathrm{C}$ (black) and $65^{\circ} \mathrm{C}$ (blue), Red - model turbid solution $\left(\mathrm{CaCO}_{3}\right)$.

Buffer: 100mM Na-phosphate, pH 7.2; Concentrations: phLDH $4 \mu \mathrm{M}, \mathrm{NADH} 5 \mu \mathrm{M}$; Arrows: light scattering peak (235 nm) and excitation wavelengths (240 nm, $285 \mathrm{~nm}$ and $350 \mathrm{~nm})$, used in our "three -in-one excitation essay" described in Methods. In fact, we used the excitation wavelength of $240 \mathrm{~nm}$ instead of $235 \mathrm{~nm}$ to measure light scattering of the samples to adjust all 3 measured parameters (FRET, 280nm/470nm; NADH fluorescence, $350 \mathrm{~nm} / 470 \mathrm{~nm}$ and light scattering, $240 \mathrm{~nm} / 470 \mathrm{~nm}$ ) to one set up of the experiment including sensitivity, slit width of the monochomators etc. 


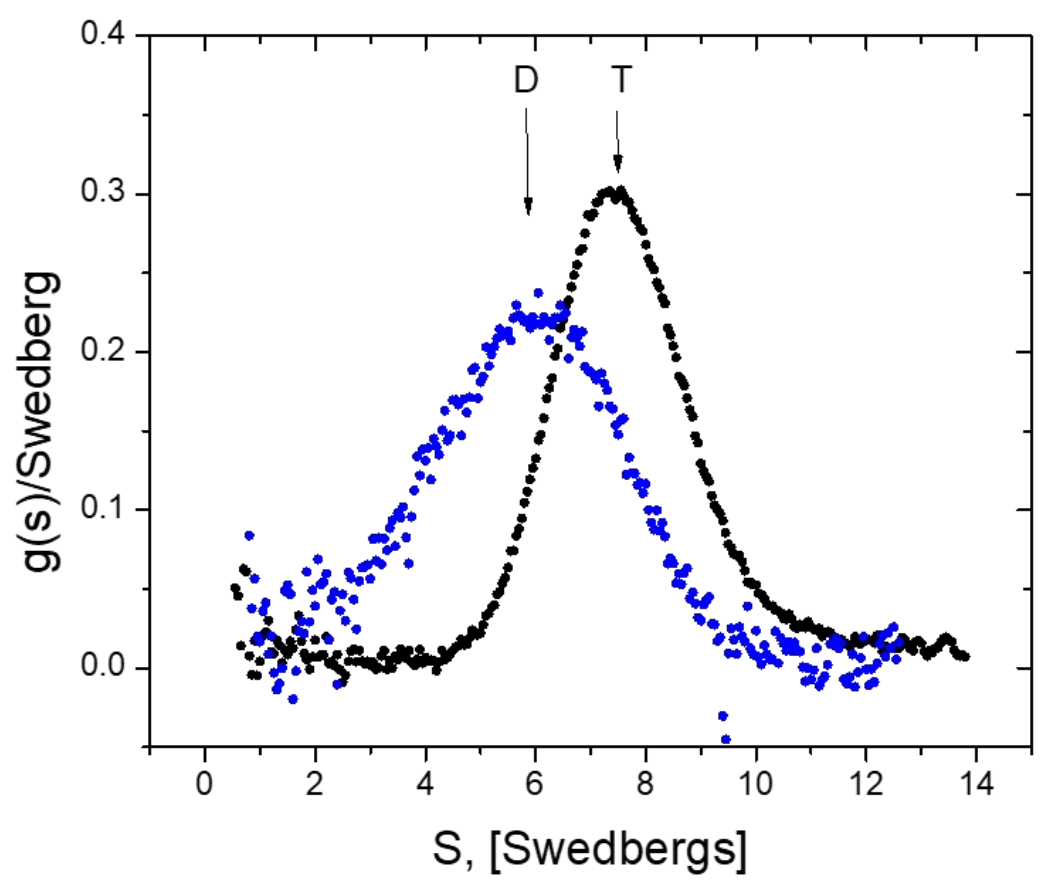

Figure S2. The size distribution of Michaelis complexes for bsLDH in the presence (T, tetramers) and the absence (D, dimers) of 5 mM FBP (D-Fructose 1,6bisphosphate).

Ultracentrifugation, bsLDH; Experiment was conducted at $20{ }^{\circ} \mathrm{C}$ following protein absorption at $292 \mathrm{~nm}$ using a Beckman XL-I analytical ultracentrifuge run at 55,000 rpm. The sedimentation boundaries are presented as time-derivative distributions calculated using the program DCDT; Buffer: 100 mM TEA (Triethanolamine $x \mathrm{HCl}$ ), $\mathrm{pH} 7.6$ 

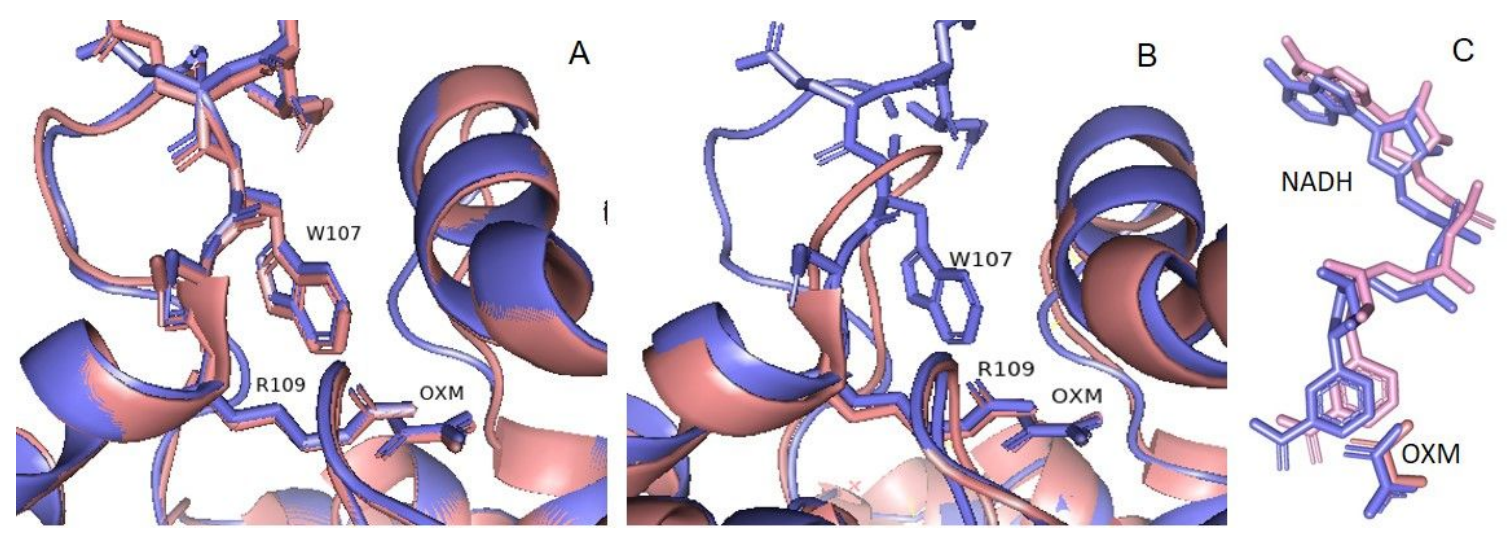

Figure S3. Structural alignment of LDH from human heart (hhLDH) and Plasmodium falciparum (pfLDH) showing the substrate specificity group (97-112) and the five inserted residues (106-110) of pfLDH in detail. AA numbering is shown according to the numbering in the crystal structure (1LDG, Dunn et al, 1996). A, overlay of crystal structures of the tertiary complexes (LDH+NADH+Oxamate) of pfLDH, wild type (1LDG, blue) and S245A, A327P mutant (1T2E, orange); $\mathrm{NADH}$ is not shown for clarity. $\mathbf{B}$, overlay of the tertiary complexes ( $\mathrm{LDH}+\mathrm{NADH}+\mathrm{Oxamate}$ ) of pfLDH, wild type (1LDG, blue) and hhLDH (1IOZ, pink), NADH is not shown for clarity. C, Superposition of the active site pockets of hhLDH (1I0Z, pink) and pfLDH (1LDG, blue) 